\title{
OBSERVATION OF A BACTERIOCHLOROPHYLL ANION RADICAL DURING THE PRIMARY CHARGE SEPARATION IN A REACTION CENTER
}

\author{
W. HOLZAPFEL a , U. FINKELE ${ }^{\text {a }}$, W. KAISER ${ }^{\text {a }}$, D. OESTERHELT ${ }^{\mathrm{b}}$, H. SCHEER ${ }^{\text {, }}$ \\ H.U. STILZ ${ }^{b}$ and W. ZINTH ${ }^{a}$ \\ " Physik Department der Technischen Universität München, D-8000 Munich, Federal Republic of Germany \\ - Max-Planck-Institut fï Biochemie, D-8033 Martinsried/Munich, Federal Republic of Germany \\ c Rotanisches Institut der Ludwig-Maximilian-Universität, D-8000 Munich, Federal Republic of Germany
}

Received 13 June 1989

\begin{abstract}
The primary light-induced charge separation in reaction centers of $R$ hodobacter sphaeroides was investigated with femtosecond time resolution. The absorption changes in the time range $100 \mathrm{fs}$ to $1 \mathrm{~ns}$ observed after direct excitation of the primary donor $P$ at $860 \mathrm{~nm}$ could only be explained by a kinetic model which uses three time constants. This finding supports the following reaction scheme: (i) the electronically excited primary donor $\mathrm{P}^{*}$ decays with a time constant of 3.5 ps and populates a very short-lived intermediate involving a reduced accessory bacteriochlorophyll molecule; (ii) with a time constant of 0.9 ps the electron is transferred to the neighboring bacteriopheophytin molecule; and (iii) from there within 200 ps to the quinone.
\end{abstract}

\section{Introduction}

The primary photochemical event in the photosynthesis of bacteriochlorophyll-containing organisms is a light-induced charge separation within a transmembrane protein complex called the reaction center (RC). Recently, the crystal structures of the reaction centers of two purple bacteria were elucidated: Rhodopseudomonas (Rps.) viridis $[1,2]$ and Rhodobacter (Rb.) sphaeroides $[3,4]$. The prosthetic groups in the transmembrane core of the reaction centers ( 4 bacteriochlorophylls, 2 bacteriopheophytins, 2 quinones, 1 iron) are arranged as two branches $\left(A, B^{\# 1}\right)$ in an approximate $C_{2}$ symmetry. Two bacteriochlorophyll molecules form the so-called special pair (P) located on the symmetry axis. Starting at the special pair, each branch consists of an accessory bacteriochlorophyll $\left(B_{A}, B_{B}\right)$, a bacteriopheophytin $\left(H_{A}, H_{B}\right)$, and a quinone $\left(Q_{A}, Q_{B}\right)$.

Before the structure of the RC was known, information on the dynamics of light-induced charge sep-

\#1 Indices A and B [5] correspond to branches $L$ and $M$ [6], respectively. Cation and anion radicals are indicated by their charges only (c.g. $\left.\mathrm{P}^{+}, \mathrm{H}^{-}\right)$. aration had been obtained $[7,8]$ : The special pair, $\mathbf{P}$, functions as a primary electron donor. On the picosecond time scale the electron is transferred to a bacteriopheophytin $\mathrm{H}$ and from there to a quinone $Q$ [9-17]. Additional experiments have shown that the electron transfer proceeds via the $\mathrm{A}$ branch $[6,8,14]$.

Presently, there exist several theoretical models to describe the primary electron transfer in the reaction centers:

(i) Most obvious from the structure is a three-step model, where the electron is transferred in sequence from the special pair $P$ to the neighboring bacteriochlorophyll $\mathrm{B}_{\mathrm{A}}$, then to the bacteriopheophytin $\mathrm{H}_{\mathrm{A}}$, and further on to the quinone $Q_{A}[18-20]$. In this model the energy of state $\mathrm{P}^{+} \mathrm{B}^{-}$must be below the energy of state $P^{*}$ and a finite occupation time of state $\mathbf{P}^{+} \mathbf{B}^{-}$is expected. The failure to demonstrate this state convincingly $[12,16,17]$ has led to the proposal of two alternative mechanisms:

(ii) In the "super-exchange" model the energy of $\mathrm{P}^{*}$ is lower than that of state $\mathrm{P}^{+} \mathrm{B}^{-}$. The latter is thus not populated. Instead, the electron immediately travels to the bacteriopheophytin $\mathbf{H}_{\mathrm{A}}$. Nevertheless, 
bacteriochlorophyll $\mathrm{B}_{\mathrm{A}}$ is functional as a virtual electron conductor $[21,22]$.

(iii) Recently, a model was discussed where $\mathbf{B}^{*}$ instead of $P^{*}$ acts as primary donor. Excitation energy transfer occurs from $P^{*}$ to $B^{*}$, which is followed by a charge separation yielding $\mathrm{B}^{+} \mathrm{H}^{-}$and a subsequent charge transfer forming $\mathrm{P}^{+} \mathrm{BH}-\mathrm{Q}$ [20].

A number of experiments on the primary reaction dynamics have been undertaken to test these models. Most recent data obtained for Rhodobacter sphaeroides (and also for $R p s$. viridis) were contradictory to model (i). Light absorption by the special pair band $(\lambda=860 \mathrm{~nm})$ populates the lowest excited electronic state $\mathrm{P}^{*}$ of $\mathrm{P}$ lasting for $2.8 \mathrm{ps}$. This information was directly deduced from the time dependence of the stimulated emission (i.e. gain) seen at the long-wavelength side of the P-absorption band $[5,12]$. Simultaneous reduction of bacteriopheophytin $\mathrm{H}$ to $\mathrm{H}^{-}$was evaluated from data measured at $545 \mathrm{~nm}[12]$, where the bacteriopheophytin $\left(\mathrm{H}_{\mathrm{A}}\right)$ absorbs. From the absorption decrease with the same time constant of $2.8 \mathrm{ps}$ and from other data it was concluded that either no intermediate electron carrying state between $\mathrm{P}^{*}$ and $\mathrm{H}^{-}$exists, or, if it exists, its lifetime must be shorter than 100 fs $[5,12]$. It should be noted, however, that in earlier experiments - not performed under optimum conditions - evidence for such an intermediate was discussed based on the finding of a rapid transient component with decay constant $\approx 1$ ps $[13,15-17]$.

We have reinvestigated the kinetics of the primary processes of $R b$. sphaeroides under optimized experimental conditions:

(i). The excitation wavelength was chosen to excite $P$ in its $860 \mathrm{~nm}$ band.

(ii) Photon exposure was kept low to excite only a small fraction of the reaction centers during each pulse.

(iii) The time resolution was better than $100 \mathrm{fs}$.

(iv) The wavelengths of the probing pulses were varied from 500 to $1000 \mathrm{~nm}$.

(v) Data were collected for delay times $\left(t_{D}\right)$ up to $1 \mathrm{~ns}$, allowing a quantitative comparison with kinetic models.

(vi) Data were obtained at highest precision at those wavelengths and delay times which were most indicative for a short-lived intermediate.

For evaluation of the experimental data we used two different kinetic models (depicted in fig. 3). Model (a) is based on three states connected by two kinetic constants and does not account for a shortlived intermediate. Model (b) connects four states by three time constants and allows for such an intermediate. The experimental results reported here clearly favor model (b) and suggest that the additional intermediate is a $\mathrm{P}^{+} \mathbf{B}^{-} \mathrm{HQ}$ state.

\section{Experimental}

Reaction centers of $R b$. sphaeroides ATCC 17023 were isolated from phototrophically grown cells essentially as described in ref. [23], except for the following modifications [24]. Crude chromatophores were solubilized in $20 \mathrm{mM}$ Tris ( $\mathrm{pH} 8.0$ ), $0.25 \%$ lauryldimethylaminoxide (LDAO), $125 \mathrm{mM} \mathrm{NaCl}, 0.5$ mM phenylmethylsulfonylfluoride (PMSF) for 45 min at room temperature in the dark and subsequently centrifuged at $144000 \mathrm{~g}, 4^{\circ} \mathrm{C}$ for $120 \mathrm{~min}$. The resulting pellet was subjected to a second solubilization step (same concentration and same buffer without PMSF). The combined reaction center containing supernatant were diluted with an equal volume of $20 \mathrm{mM}$ Tris ( $\mathrm{pH} \mathrm{8.0)}$ ) containing 0.25 LDAO and directly applied to a DEAE 52 anion-exchange cellulose column. The column was then washed with increasing concentrations of $\mathrm{NaCl}(80,110,130,155$ $\mathrm{mM}$ ) in $20 \mathrm{mM}$ Tris ( $\mathrm{pH} 8.0$ ) containing $0.08 \%$ LDAO. Pure reaction centers were eluted at $185 \mathrm{mM}$ $\mathrm{NaCl}$. The reaction center solution was dialyzed against $20 \mathrm{mM}$ Tris ( $\mathrm{pH} 8.0$ ) containing $0.08 \%$ LDAO and afterwards concentrated to $78 \mu \mathrm{M}$ by ultrafiltration. Reaction centers from the carotenoid-less mutant $R b$. sphaeroides R26.1 were prepared with LDAO and then transferred to tris-buffer (10 mM, pH 8 ) containing Triton X-100 (1.9 mM) as previously described [25]. The samples were used directly in the spectroscopic experiments or after appropriate dilution. Measurements were done at $24^{\circ} \mathrm{C}$ in cuvettes with $1 \mathrm{~mm}$ path length and under stirring.

A synchronously pumped unidirectional ring dye laser (UDR) [26] generated pulses of duration 60 fs at a wavelength of $860 \mathrm{~nm}$. A three-stage dye amplifier increased the energy of single pulses to $20 \mu \mathrm{J}$ (repetition rate $10 \mathrm{~Hz}$ ). For excitation and probing each of these pulses was split into two parts. The ex- 
citation pulse $(0.3 \mu \mathrm{J})$ was focused to a spot diameter of $0.5 \mathrm{~mm}$, providing an energy density of $150 \mu \mathrm{J} / \mathrm{cm}^{2}$ in the sample. This photon exposure excited approximately $13 \%$ of the reaction centers. The exciting pulse had a band width of $10 \mathrm{~nm}$. At wavelengths below $840 \mathrm{~nm}$ no spectral components of the exciting pulses were detected. Consequently, there was no direct excitation of $B$ (or $\mathbf{H}$ ) via their absorption bands at $\lambda<840 \mathrm{~nm}$. The probing pulse passed an adjustable delay line and was then focused onto a thin jet of ethylene glycol to generate a femtosecond light continuum. A $10-18 \mathrm{~nm}$ wide portion of this continuum was selected by means of a special dispersion compensating spectrometer [27]. The energy density of the probing pulse was less than 7 $\mu \mathrm{J} / \mathrm{cm}^{2}$. Exciting and probing pulses were polarized parallel and for each probing wavelength $\lambda_{\mathrm{pr}}$ the experimental response function was recorded. Typical values of $130 \mathrm{fs}$ were obtained, allowing the determination of time constants somewhat below $100 \mathrm{fs}$.

The weak photon exposure necessarily caused only small changes of transmission $(<2 \%)$ in the samples. These small absorption changes could be accurately measured by a sensitive difference detection system [28]. For each delay time $t_{\mathrm{D}}$ the average of at least 1000 absorption measurements was calculated.

\section{Results}

The inset of fig. 1 depicts the absorption spectrum of the reaction centers of $R b$. sphaeroides R26.1. The broad absorption band at $860 \mathrm{~nm}$ is due to the electronic transition of lowest energy in the special pair $P$ ( $Q$, transition) and irradiation in that range ascertains selective excitation. The $\mathrm{Q}_{y}$ transitions of the other pigments occur at shorter wavelengths, at 805 (mainly due to the two bacteriochlorophylls B) and $760 \mathrm{~nm}$ (bacteriopheophytins $\mathrm{H}$ ); the corresponding $Q_{x}$ transitions occur at 600 (P and $B$ ) and $540 \mathrm{~nm}(\mathrm{H})$.

Time-resolved absorption changes are plotted in figs. 1 and 2 as a function of delay times between -1 and +1 ps on a linear scale and from 1 ps to 1 ns on a logarithmic scale. The experimental data points are shown as full circles. The curves are calculated according to the kinetic model (a) (three components,

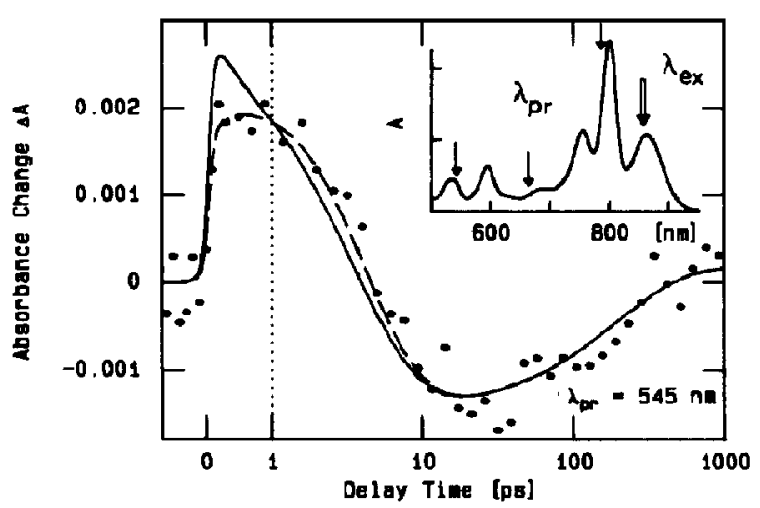

Fig. 1. Time-resolved absorption data (full circles) taken at a probing wavelength of $454 \mathrm{~nm}$ for reaction centers of $R b$. sphaeroides $\mathrm{R} 26.1$ (excitation wavelength $860 \mathrm{~nm}$ ). The delay time scale is linear between -1 and $+1 \mathrm{ps}$ and logarithmic for $t_{\mathrm{D}}>1$ ps. The curves are calculated according to two models presented in fig. 3 and discussed in the text. The inset depicts the absorption spectrum of the sample.

solid line) and (b) (four components, dashed line).

The results in fig. 1 were obtained for a probing wavelength of $\lambda_{\mathrm{pr}}=545 \mathrm{~nm}$. At this wavelength the bacteriopheophytin $\mathbf{H}_{\mathrm{A}}$ absorbs (see inset) and absorption changes include those due to changes of the redox state of $\mathbf{H}_{A}$. First, the absorption increases instantaneously with the excitation pulse due to formation of $\mathrm{P}^{*}$. In the time domain of 1 to $10 \mathrm{ps}$ an absorption decrease is observed, which is reversed with a time constant of 200 ps. The three-component model (a) (on the basis of the time constants 3.5 and $200 \mathrm{ps}$ ) yields the time course shown by the solid line. It correctly describes the experimental data at late delay times $t_{\mathrm{D}}>10 \mathrm{ps}$. At shorter times, however, a systematic difference between experimental data points and the predicted curve is seen which cannot be eliminated for any choice of time constants. Only the introduction of a fourth kinetic component with a time constant of 0.9 ps gives satisfactory agreement between data points and calculated curve (dashed line).

Strong evidence for the existence of an additional intermediate is also obtained from data taken at other probing wavelengths. In figs. $2 a$ and $2 b$ data are shown for probing wavelengths of 785 and $665 \mathrm{~nm}$. At $785 \mathrm{~nm}$, where the bacteriochlorophylls and the bacteriopheophytins absorb (fig. 2a), positive ab- 


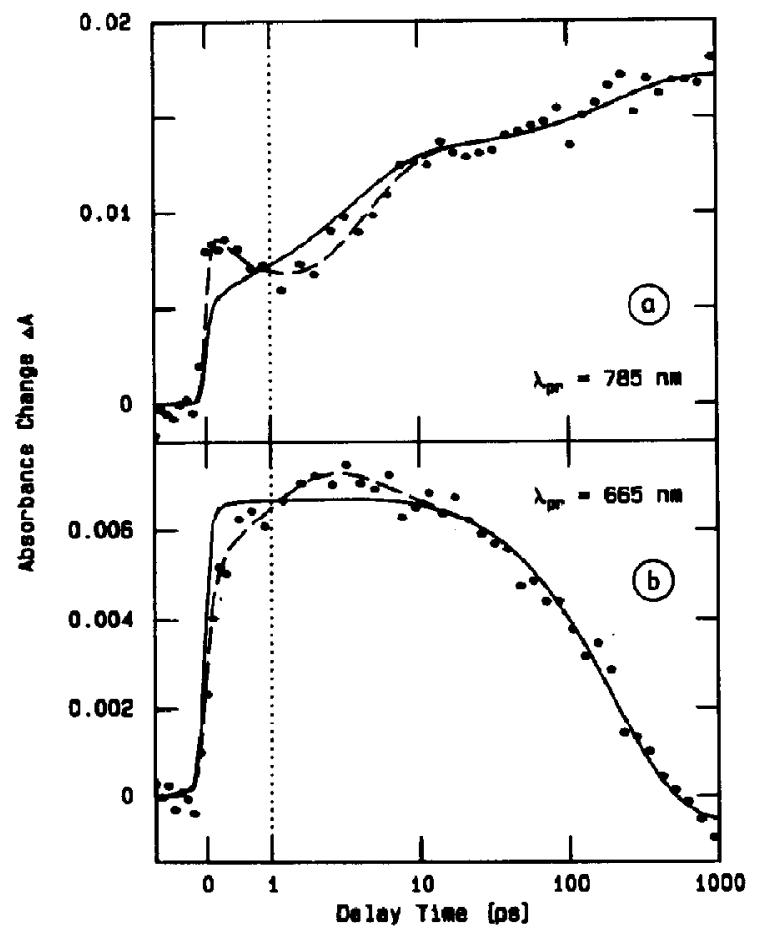

Fig. 2. Time-resolved absorption data (full circles) for probing wavelengths of $785 \mathrm{~nm}$ (a) and $665 \mathrm{~nm}$ (b) for reaction centers of wild-type $R b$. sphaeroides ATCC 17023 (excitation $860 \mathrm{~nm}$ ). The curves are calculated for the two models of fig. 3 .

sorption changes are found throughout the time range analyzed: The first rapid rise of absorption follows the excitation pulse to a first distinct maximum at $300 \mathrm{fs}$. Then absorption decreases until $t_{\mathrm{D}}=1.2 \mathrm{ps}$. A subsequent absorption increase proceeds in the 3 ps time domain, followed by a second slow increase in the $200 \mathrm{ps}$ range. The solid curve represents the absorption change predicted by the three-component model (a) and clearly deviates from the experimental values in the time range of zero to $10 \mathrm{ps}$. The fourcomponent model (b) on the other hand, fits the experimental data quite well, in particular during the first picosecond (dashed line).

The experimental results of fig. $2 b$ obtained with $\lambda_{\mathrm{pr}}=665 \mathrm{~nm}$ present the spectral range where the reduced bacteriochlorophylls and the reduced bacteriopheophytins absorb [30]. Once more, the results confirm the existence of the 0.9 ps component. At this wavelength the instantaneous rise of absorption is followed by a second and slower increase. At later times absorption decreases with time constants of 3.5 and 200 ps. Again a least-squares fit of the threecomponent model (a) (solid line) does not satisfactorily explain the data between 0.2 and $10 \mathrm{ps,}$ while the four-component model (b) is in full agreement (dashed line).

Additional results - not documented in this Letter - should be briefly reported:

(a) Transient changes of absorption at probing wavelengths of $690 \mathrm{~nm}$, and between 760 and 800 $\mathrm{nm}$, confirm the existence of an intermediate with a time constant of 0.9 ps. This component is not seen in the $Q_{y}$ absorption and gain range of the special pair between 860 and $970 \mathrm{~nm}$. Here the absorption changes decay monoexponentially.

(b) Experiments $\left(\lambda_{\mathrm{pr}}=860 \mathrm{~nm}\right)$ have been performed at a photon exposure reduced by a factor of three. Under these conditions only $4 \%$ of the RCs are excited by the laser pulse. The same absorption characteristics including the $0.9 \mathrm{ps}$ component were found.

(c) Three different samples, RC from wild-type $R b$. sphaeroides ATCC 17023, wild-type RC with sodium ascorbate added $(0.2 \mathrm{mM})$, and $R C$ from the carotenoid-deficient strain $R 26$, were compared. For all samples the $0.9 \mathrm{ps}$ component is required to explain the observed absorption changes.

\section{Discussion}

A theoretical description of the time-resolved absorption data consists of rate equations for the population of molecular states $I_{j}$. The first state $I_{1}$ is populated by optical excitation. Sequential relaxation with time constants $\tau_{j}$ leads to daughter states (see fig. 3) ${ }^{\# 2}$. It can be shown that the absorption change $\Delta A\left(t_{\mathrm{D}}\right)$ found under these conditions is a sum of single-sided exponentials with time constants $\tau_{j}$ convolved with the experimental response function [31]. Comparison of the calculated curve and the experimental data allows the determination of the amplitudes of the various components, i.e. the difference of absorption cross-sections as well as the decay time constants of the individual states. At some probing

\#2 An unbranched reaction scheme is assumed here. 

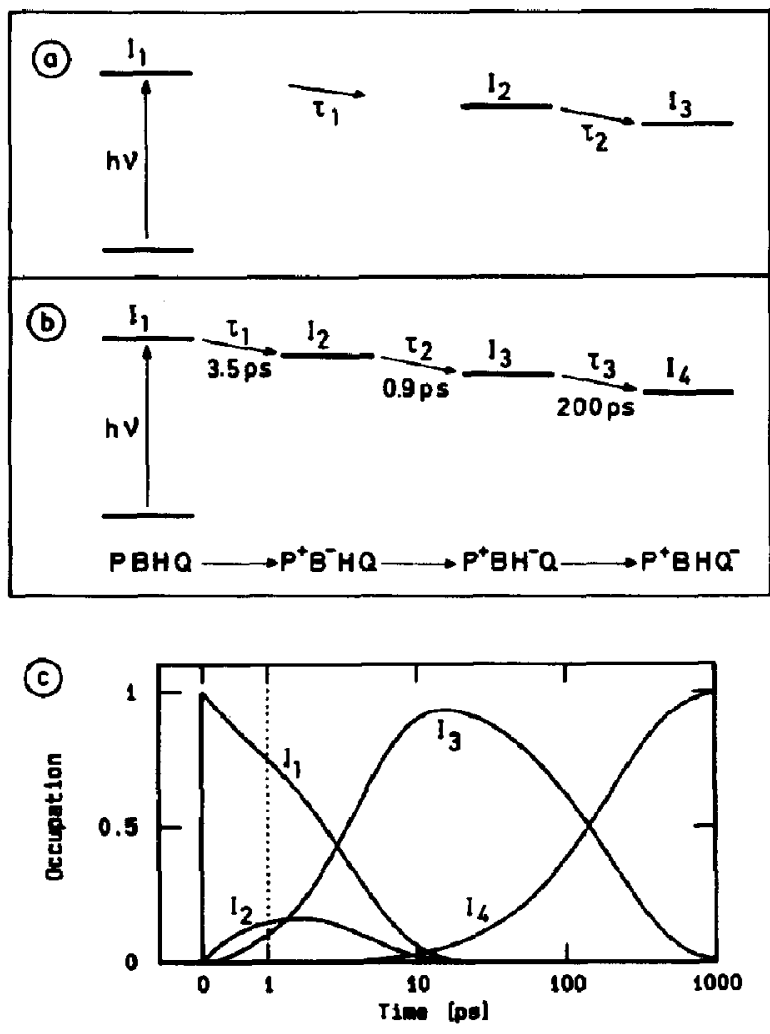

Fig. 3. (a, b) Schemes of the sequence of states considered as alternatives for electron transfer in reaction centers. Models in (a) and (b) refer to the solid and dashed lines of the absorbance changes in figs. 1 and 2, respectively. (c) Population densities of the states $I_{i}$ as functions of time calculated according to model (b). Note that the population of $\mathrm{I}_{2}\left(\mathrm{P}^{+} \mathrm{B}-\mathrm{HQ}\right)$ remains smaller than $16 \%$ due to slow population (3.5 ps) and fast depopulation ( $0.9 \mathrm{ps}$ ) of this state.

wavelengths individual kinetic components may not be apparent. We note that the shortest time constant is always seen at the beginning of the time-resolved measurements, irrespective of its location in the reaction sequence.

We treated two different models for comparison with experimental data. The first simpler model (a) (from which solid curves in figs. 1 and 2 were derived) describes electron transfer as involving three states $I_{t}$ to $I_{3}$ which are connected by two time constants, $\tau_{1}=3.5$ and $\tau_{2}=200$ ps (fig. $3 \mathrm{a}$ ). The second model (b) uses four states, $I_{1}$ to $I_{4}$, and three relax- ational processes with time constants $\tau_{1}=3.5, \tau_{2}=0.9$, $\tau_{3}=200$ ps (see fig. $3 b$ ).

The first state $I_{1}$ to be considered is the excited electronic state $\mathrm{P}^{*}$. It is observed in the wavelength range 860 to $970 \mathrm{~nm}$, where only a monoexponential absorption change with a time constant of $3.5 \pm 0.4$ ps is found. As a consequence, this time constant must be the first one $\left(\tau_{1}\right)$ in the sequence of model (a) and model (b). Our value of the decay time of $P^{*}$ is somewhat larger than the $2.8 \pm 0.2$ ps published previously [12].

The intermediates of the electron transfer process are seen at shorter wavelengths, for instance at 545 , 665 , and $785 \mathrm{~nm}$. At these wavelengths the experimental data at early delay times are not well accounted for by the prediction of the three-component model (a) (solid lines) but strongly suggest a fourth component. The corresponding time constant is short, with a value of $0.9 \pm 0.3 \mathrm{ps}$. The analysis of the time-resolved absorption data - the time dependence and the absorption amplitudes at various probing wavelengths - indicate that the molecular intermediate related to the additional component must occur after the 3.5 ps relaxation of $P^{*}$ and prior to the $200 \mathrm{ps}$ process leading to a reduced quinone. In the framework of a sequential model this result leads to the conclusion that an additional intermediate $I_{2}$ is required. The most obvious candidate is the configuration $\mathrm{P}^{+} \mathrm{B}^{-} \mathrm{HQ}$ containing a bacteriochlorophyll anion radical. This interpretation is supported by the following observations:

(i) $I_{2}$ shows strongly reduced absorption at the wavelength $797 \mathrm{~nm}$, where the accessory bacteriochlorophyll $B$ absorbs $\left(Q_{y}\right.$ band) and where $B^{-}$has a strongly reduced absorption [30].

(ii) During the rapid decay of $\mathrm{I}_{2}$ the absorption recovers.

(iii) The relatively slow population ( $3.5 \mathrm{ps})$ and faster depopulation ( $0.9 \mathrm{ps})$ of the state $\mathrm{B}^{-}$gives rise to a small transient population of $16 \%$ only (see fig. $3 \mathrm{c})$. This small concentration of $\mathrm{B}^{-}$makes the experimental observation a non-trivial task.

(iv) $I_{2}$ does not show absorption decrease in the wavelength range where bacteriopheophytin absorbs (e.g. at $454 \mathrm{~nm}$ ).

State $I_{3}$ represents the next intermediate where the electron has reached the bacteriopheophytin $H$ forming the configuration $\mathrm{P}^{+} \mathrm{BH}^{-} \mathrm{Q}$. The related de- 
crease of the $\mathrm{H}$ absorption at $545 \mathrm{~nm}$ is clearly seen after the decay of state $I_{2}$ and prior to the formation of $I_{4}$ (see fig. 1). Finally, in state $I_{4}$ the electron arrives at the quinone $Q_{A}$. The remaining absorption changes are due to the configuration $\mathrm{P}^{+} \mathrm{BHQ}^{-}$.

At this point, previously reported data should be mentioned. Several authors have reported the observation of a $1 \mathrm{ps}$ component $[12,13,15,17]$ after femtosecond excitation of RC at wavelengths around $610 \mathrm{~nm}$. Since this excitation wavelength does not start the charge separation process at the lowest electronic level of the primary donor $\mathbf{P}$, the data were disregarded as evidence for an additional intermediate in the electron-transfer process.

Experiments performed at the favorable excitation wavelength around $860 \mathrm{~nm}$ but with pulse durations of $30 \mathrm{ps}$ reported a short-lived component preceding the bacteriopheophytin $[16,17]$. Unfortunately, the long pulses and limited time resolution did not permit firm conclusions.

Detailed investigations with femtosccond pulses at the optimum excitation wavelength of $860 \mathrm{~nm}$ were reported on RC from $R b$. sphaeroides [12]. Some of these experiments done with probing wavelengths of 860 to $900 \mathrm{~nm}$ clearly showed the monoexponential decay of P*. But the data on the formation of the first ground-state species contained substantial "statistical noise". These experiments were not able to rule out a weak (15-20\%) contribution of an additional short-lived intermediate [18].

We have performed a detailed experimental study of the primary charge transfer processes in the reaction centers of $R b$. sphaeroides. The analysis of the kinetic data with a linear model strongly suggests that the primary charge transfer to the bacteriopheophytin $\mathbf{H}$ proceeds via the accessory bacteriochlorophyll $B$ as a true intermediate. The following reaction scheme results: After excitation of the special pair $P$ an electron is transferred with a time constant of 3.5 ps to the accessory bacteriochlorophyll $B$. Here the electron resides for the short time of $0.9 \mathrm{ps}$ before it reduces the bacteriopheophytin $\mathrm{H}$.

\section{Acknowledgement}

This work was supported by the Deutsche Forschungsgemeinschaft SFB 143. HS thanks Dr. G.
Reng (Gesellschaft für Biotechnologische Forschung, D-3301 Stockheim) for the mass culture of Rhodobacter sphaeroides R26.1, and acknowledges the skillful technical assistance of $\mathrm{C}$. Bubenzer.

\section{References}

[1] J. Deisenhofer, O. Epp, K. Miki, R. Huber and H. Michel, J. Mol. Biol. 180 ( 1984 ) 385.

[2] H. Michel, O. Epp and J. Deisenhofer, EMBO J. 5 (1986) 2445;

J. Deisenhofer and H. Michel, in: The photosynthetic bacterial reaction center - structure and dynamics", eds. J. Breton and H. Vermeglio (Plenum Press, New York, 1988) pp. 1-3.

[3] J.P. Allen, G. Feher, T.O. Yeates, H. Komiya and D.C. Rees, Proc. Natl. Acad. Sci. US 84 (1987) 5730.

[4] C.H. Chang, D. Tiede, J. Tang, U. Smith, J. Norris and M. Schiffer, FEBS Letters 205 (1986) 82.

[5] J. Breton, J.-L. Martin, A. Migus, A. Antonetti and A. Orszag, Proc. Natl. Acad. Sci. US 83 (1986) 5121.

[6] W. Zinth, E.W. Knapp, S.F. Fischer, W. Kaiser, J. Deisenhofer and H. Michel, Chem. Phys. letters 119 (1985) 1.

[7] W.W. Parson, Ann. Rev. Biophys. Bioeng. 11 (1982) 57.

[8] R.M. Hochstrasser and C.K. Johnson, in: Topics in applied physics, Vol. 60. Ultrashort laser pulses and applications, ed. W. Kaiser (Springer, Berlin, 1988) p. 357.

[9] K.J. Kaufmann, P.L. Dutton, T.L. Netzel, J.S. Leigh and P.M. Rentzepis, Science 188 (1975) 1301.

[10] K.J. Kaufmann, K.M. Petty, P.L. Dutton and P.M. Rentzepis, Biochm. Biophys. Res. Commun. 70 (1976) 839.

[11] D. Holten, M.W. Windsor, W.W. Parson and J.P. Thornber, Biochim. Biophys. Acta 501 (1978) 112.

[12] J.-L. Martin, J. Breton, A.J. Hoff, A. Migus and A. Antonetti, Proc. Natl. Acad. Sci. US 83 (1986) 957;

G.R. Fleming, J.-L. Martin and J. Breton, Nature 333 (1988) 190.

[13] D. Holten, C. Hoganson, M.W. Windsor, C.C. Schenck, W.W. Parson, A. Migus, R.L. Fork and C.V. Shank, Biochim. Biophys. Acta 592 (1980) 461.

[14] C. Kirmaier, D. Holten and W.W. Parson, Biochim. Biophys. Acta 810 (1985) 33 .

[15] W. Zinth, M.C. Nuss, M.A. Franz, W. Kaiser and H. Michel, in: Springer series in chemical physics, Vol. 42. Antennas and reaction centers of photosynthetic bacteria, ed. M.E. Michel-Beyerle (Springer, Berlin, 1985) p. 286.

[16] V.A. Shuvalov and A.V. Klevanik, FEBS Letters 160 (1983) 51.

[17] V.A. Shuvalov, J. Amesz and L.N.M. Duysens, Biochim. Biophys. Acta 851 (1986) 327.

[18] R.A. Marcus, Biochim. Biophys. Acta 811 (1985) 265; Chem. Phys. Letters 133 (1987) 471. 
[19] W.W. Parson, A. Warshel, C. Creighton and J.R. Norris, in: The photosynthetic bacterial reaction center - structure and dynamics, eds. J. Breton and H. Vermeglio (Plenum Press, New York, 1988 ) p. 309.

[20] S.F. Fischer and P.O.J. Scherer, Chem. Phys. 115 (1987) 151 .

[21] M. Bixon, J. Jortner, M. Plato and M.E. Michel-Beyerle, in: The photosynthetic bacterial reaction center - structure and dynamics, eds. J. Breton and H. Vermeglio (Penum Press, New York, 1988) p. 399.

[22] J. Jortner and M.E. Michel-Beyerele, in: Springer series in chemical physics, Vol. 42. Antennas and reaction centers of photosynthetic bacteria, ed. M.E. Michel-Beyerle (Springer, Berlin, 1985) p. 344.

[23] A. Ogrondnik, M. Volk, R. Letterer, R. Feick and M.E. Michel-Beyerle, Biochim. Biophys. Acta 936 (1988) 361.
[24] R. Feick, private communication.

[25] D. Beese, R. Steiner, H. Scheer, A. Angerhofer, B. Robert and M. Lutz, Photochem. Photobiol. 46 (1987) 293.

[26] J. Dobler, H.H. Schulz and W. Zinth, Opt. Commun. 57 (1986) 407.

[27] O.E. Martinez, IEEE J. Quantum Electron QE-23 (1987) 59.

[28] H.J. Polland and W. Zinth, J. Phys. E 18 (1985) 399.

[29] J. Breton, J.-L. Martin, G.R. Fleming and J.-C. Lambry, Biochemistry 27 (1988) 8276.

[30] M.S. Davis, A. Forman, L.K. Hanson, J.P. Thornber and J. Fajer, J. Phys. Chem. 83 (1979) 3325.

[31] H.J. Polland, M.A. Franz, W. Zinth, W. Kaiser, E. Kolling and D. Oesterhelt, Biophys. J, 49 (1986) 651. 